\title{
Bargaining Solutions for Energy Efficient and Fair Power Allocation in Cognitive D2D Communications
}

\author{
Sonia Fourati \\ Mediatron Lab. \\ Communication Engineering \\ School (Sup'Com), Tunisia
}

\author{
Soumaya Hamouda \\ Mediatron Lab. \\ Communication Engineering \\ School (Sup'Com), Tunisia
}

\author{
B. T. Maharaj \\ Department of EECE, \\ University of Pretoria, \\ South Africa
}

\begin{abstract}
Device-to-Device (D2D) technology underlying the cellular network is an attractive solution for future generation network to increase cellular traffic offloading. In order to reduce the interference caused to cellular network, D2D users can opportunistically access the cellular spectrum using cognitive radio capabilities. They can either avoid interference by transmitting on the spectrum wholes or merely control their power while transmitting simultaneously with the cellular users. In this paper, cognitive D2D users, referred to as Secondary Users (SUs), communicate at the same time as uplink cellular users. A new power control approach is proposed based on bargaining game theoretic solutions to better control SUs' transmit power and thus reduce the interference level at the cellular base station referred to as the Primary User (PU). First, the SUs utility functions are defined and take into account the achieved data rate, the power consumption and the impact of the interference. Then, the optimal power allocation is analyzed through the application of the Nash bargaining, Kalai-Smorodinsky and other bargaining solutions. A comparison between the performance of these solutions in terms of energy efficiency and fairness is derived. Simulation results show that a tradeoff between fairness and energy efficiency should be taken into account. The performance of cooperative solutions is also compared with non-cooperative games both analytically and through simulations.
\end{abstract}

\section{General Terms}

Cognitive radio, Game Theory, D2D Communications, 5G.

\section{Keywords}

Cognitive Device-to-device; power allocation; game theory; NBS; KSBS; energy efficiency; fairness

\section{INTRODUCTION}

The increasing demand for wireless multimedia services has lead to increasingly crowded spectrum, and a large amount of data traffic is generated from mobile devices (eg. video streaming, video gaming, Internet of Things IoT...). Cognitive radio (CR) technology is considered as a promising technology to improve the spectrum utilization and spectrum efficiency [1]. It adopts the spectrum sharing technology to make the Secondary Users (SUs) access opportunistically the licensed band used by the Primary Users (PUs). However, the PUs still have the priority to utilize this band. SUs can coexist with the PUs in an underlay mode and must control his transmit power to keep the interference level below a certain threshold, named interference temperature limit (ITL) [1].

Similarly to Cognitive Radio Networks (CRNs), Device-todevice (D2D) has been recently proposed as a promising technique to improve resource utilization in cellular networks by offloading the mobile data traffic through the BS to local direct links among devices [2]. A D2D communication is a direct link from D2D transmitter to D2D receiver underlying a cellular infrastructure [3][4] under the control of the cellular network. The D2D communication allows peer-to-peer transmission among mobile devices bypassing the use of the evolved Node B (eNB) in the LTE network. The deployment of D2D communication certainly ensures an interesting traffic offloading for operators but faces several challenges as interference can be caused to cellular users while sharing the same radio resources. CRNs and D2D received significant research due to their incorporation to the future releases of LTE-Advanced (LTE-A) [3][5] and 5G cellular systems [6]. D2D terminals can indeed have cognitive capabilities to access the cellular network spectrum dynamically and in an opportunistic manner. In this paper, it is assumed that these terminals deploy cognitive radio underlay mode. Therefore, D2D terminals, which are considered as Secondary Users (SUs), are expected to communicate with their receivers in proximity, transmit on the cellular network bandwidth and execute power control to ovoid interference to cellular's terminals transmissions. Since SUs operate on the network's uplink band, the Primary Users (PUs) correspond to the different eNBs in the LTE-A network.

On the other hand, cooperative communication has shown great advantages to achieve higher data rate of future wireless networks while satisfying the quality of service. In this paper, performance analysis of different cooperation strategies between D2D terminals is proposed to improve the Energy Efficiency (EE) as well as fairness among them. In this comparison, a new utility function is introduced which takes into account the achieved data rate, the power consumption and the interference impact.

The rest of the paper is organized as follows. Section II presents some related work which summarizes the main previous contributions on power control strategies in D2D and cognitive radio networks. Section III describes the system model. In Section IV, a new non-cooperative game is proposed and the Nash Equilibrium state is derived. In Section V, our different bargaining solutions is presented based on the former proposed SU payoff, and the Nash bargaining problem is solved by using convex optimization techniques. Simulation results are given and commented in section VI. Section VII concludes the paper.

\section{RELATED WORK}

Many researches were conducted to resolve the power control problem in CRNs. More recently, other contributions on this issue in D2D communications have appeared using the benefits of CRNs. This section studies the way that the power allocation problem was handled first in CRNs and then in the context of D2D communications. 
CR terminals are autonomous and smart enough to learn from their environment and optimize their performance by adapting their transmission parameters. Therefore, their interactions can be modeled using Game Theory. In every game model, the SUs are the players and their strategies are the selection of new transmission parameters which influences their own performance as well as the performance of their neighboring players. The desired performance is designed as the payoff function. Game theory has been widely applied to design and analyze distributed resource allocation algorithms for CR networks (e.g. [7][8]) and D2D communications [9][10].

Many proposed power control games in CRNs are noncooperative games, such [7][8][11]. In these games, selfish SUs maximize their individual utilities in a self-interested manner without being concerned with the impact of their strategies on other users. In [7], the authors presented a noncooperative game approach to minimize the overall transmit power under each user's power limitation and minimal rate constraint. The authors defined a power based utility function in [8] and proposed a non-cooperative power game with pricing to give a Pareto improvement over the NE. In [11], a game is employed to perform power allocation in an OFDM CRN. Such power allocation is aimed to the up-link communication toward the CR local base station. A game theoretic solution for distributed uplink resource allocation in multi-cell OFDMA systems is presented in [12] where convergence and Nash equilibrium was proved using potential game. The potential games are introduced because they can guarantee the existence of at least one NE.

Non-cooperative game theory studies the strategic choice of each player independently for improving its own performance. Unfortunately, the NE has been proven to be inefficient [4], which means that the achievable network-wide sum utility can be low. For this reason, there has been many recent works that aim to improve the overall network utility through cooperative games. In CRNs, SUs are motivated to cooperate with one another to enhance their own transmission opportunities and achieve high spectrum efficiency. As a result, cooperative game theory is preferred for distributed power control [7]. In fact, efficiency and fairness are two of the most concerned metrics for power control algorithms, both of which can be handled well by the cooperative game property arising from the Nash axioms ensures user fairness [13].

On the other hand, authors in [14] propose a new Nash bargaining solution (NBS) for joint channel and power allocation in OFDMA cognitive radio (CR) systems with the objective of maximizing the overall throughput of the CR system with the protection of PUs' transmission. In [2], author studies the fairness among the primary users (PUs) and the secondary users (SUs) for resource allocation in cognitive radio systems. In [13], the authors studied the system efficiency and the fairness among the PUs and the SUs based on kalai-Smorodinsky bargaining solution compared with the sum-rate maximization and the Max-Min rate. In [15], the authors proposed a cooperative Nash Bargaining Power Control Game (NBPCG), where interference power constraints (IPCs) are imposed to protect the primary users' (PUs) transmissions, and minimum signal-to-interference plus-noise ratio (SINR) requirements are employed to provide reliable transmission opportunities to SUs. An SINR-based utility function is designed for this game model, which may reflect the spectrum efficiency of the CRN. Simulations showed the effectiveness of this NBPCG algorithm for efficient and fair power control in CRNs.
Several other recent works have considered the use of game theory in the D2D communications. The D2D devices can compete or cooperate with each other to reuse the radio resources in D2D networks. Therefore, resource allocation and access for D2D communication can be treated as games [16]. The author in [4] integrates D2D communication into LTE-Advanced networks and studied the problem of power control for D2D users (UEs) in order to restrict the co-channel interference to cellular UEs. In [5], the author considers how to efficiently employ D2D communications for SUs in a cognitive cellular network based on Game Theory. The problem of power control for D2D users using games has been studied also in the literature [17][18][19]. The authors in [17] provided power allocation for D2D users using noncooperative game, whereas the author in [18] considers the channel allocation for D2D users through a Stackelberg game where cellular users are the leaders and D2D users are the followers. The author in [19] considered the applications of game-theoretic models to study the radio resource allocation issues in D2D communication. Also, [20] provided an analysis on optimum resource allocation and power control between the cellular and D2D connections

\section{SYSTEM MODEL}

In this section, a cellular network operating where cellular users and D2D users operate on the same bandwidth is considered. It is also assumed that all D2D users have cognitive radio capabilities and are thus considered as SUs. Since the transmission of these terminals is affecting the uplink cellular communications, the PUs are identified as the eNBs in the network. The co-existence of $M$ PUs and $N$ SUs in the resulting CRN is assumed. Figure 1 illustrates an example of D2D deployment in the network in which one eNB centered in the cell area serves the whole cell as one sector and several D2D and cellular users are randomly distributed in the cell. In this case, several SUs coexist with only one PU.

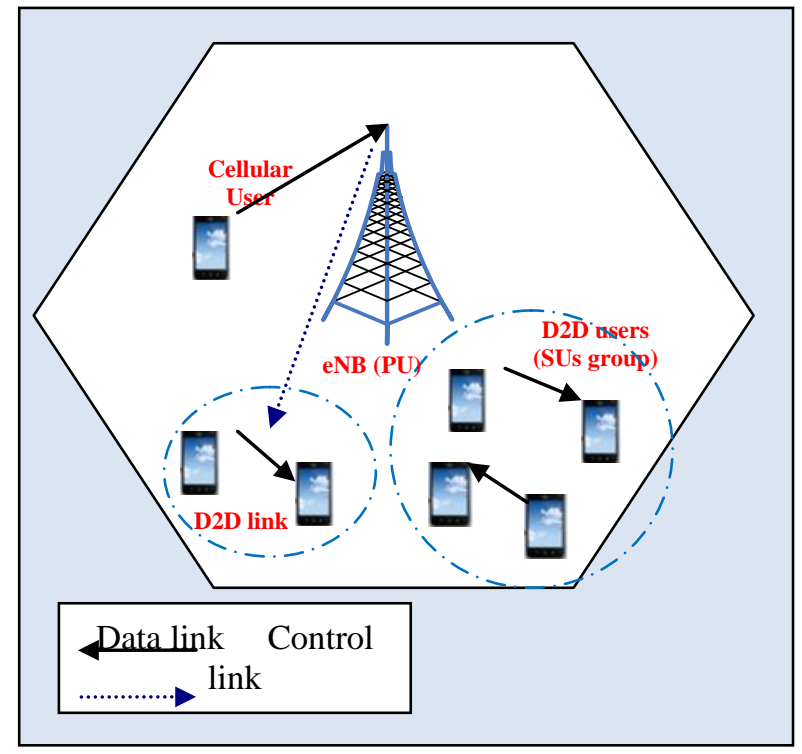

Figure 1: Layout of the cognitive D2D network

Each pair of SUs can form a D2D group $\left(v_{i}, v_{j}\right) \in \mathrm{V}$ within mutual communication range. The D2D device uses direct link to communicate with each other with operator controlled link establishment. For simplicity, the interference from others PUs (i.e. eNBs) is neglected. Thus, the signal-tointerference-and-noise ratio (SINR) between the SU 
transmitter (i.e. D2D Tx user) $i$ and the SU receiver (i.e. D2D $\mathrm{Rx}$ user) $j$ can be written as:

$$
\gamma_{i, j}=\frac{h_{i j} p_{i}}{\sum_{k=1, k \neq j}^{N} h_{k j} p_{k}+\sum_{m=1}^{M} h_{m j} p_{m}+\sigma^{2}}
$$

where $h_{i j}$ is the channel gain. The $p_{i}$ is the SU $i$ transmit power to the $\mathrm{SU} j, p_{k}$ is the transmit power of other SU different $k$ and $p_{m}$ is the transmit power of the PUs $m$ and $\sigma^{2}$ is the additive white Gaussian Noise power. Then the transmission rate of the node $i$ at time $t$ is:

$$
R_{i}=W \log _{2}\left(1+\gamma_{i j}\right)
$$

where $W$ is the channel bandwidth.

\section{PROPOSED NON-COOPERATIVE GAME: PAYOFF DESIGN AND CONVERGENCE TO NASH EQUILIBRIUM \\ 4.1 The proposed non-cooperative game design}

Game Theory based power allocation approach in the secondary network implies the definition of a proper framework. Indeed, the defined game must lead to a stable equilibrium point called Nash Equilibrium (NE). Such framework needs the definition of the players to be involved in the game as well as a set of possible strategies. In this study, the players are represented by the $N$ SUs. The strategies are the actions that each player can choose in order to adapt to the opponent player's choices. The amounts of power are considered as the possible strategies for the players SUs. Finally, the utility function should not only reflect the network spectrum utilization efficiency but also facilitate the implementation of power control algorithms in terms of convexity and global convergence. The key is to find utility expressions that are not only physically meaningful for D2D communications but mathematically attractive for ensuring global convergence to the NE as well. In this paper, the SINR in eq (1) is adopted as the QoS metric for SU players and accordingly construct the utility function $U_{i}$ in an SINRrelated form. The utility function represents the future benefit that a player will achieve when applying a certain power allocation. In this case, the utility function maps the power allocation strategy for the $i^{\text {th }}$ player into a real number while considering also the power allocated by other terminals. Therefore, the utility function for the $i^{\text {th }}$ cognitive player is defined as follows:

$U_{i}\left(p_{i}, p_{-i}\right)=N \log \left(R_{i}\right)-\beta p_{i}-\alpha \sum_{i=1}^{N} h_{i m} p_{i}$

where $R_{i}$ represents the rate that the SU $i$ achieves and $N$ is the number of SUs. The $\beta p_{i}$ is the cost of allocated power to SU $i$, the sum of $h_{i m} p_{i}$ in the second part of (3) considers the interference caused on the PU $m$.

Consequently, each player attempt to selfishly maximize his own payoff without considering the other players payoffs. This power allocation problem can thus be solved to find the Nash equilibrium (NE) as follows:

$$
\max _{p_{i}} U_{i}\left(p_{i}, p_{-i}\right)
$$

$$
\begin{array}{ll}
\text { s.t. } & \gamma_{i} \geq \gamma_{\min } \\
& \sum_{i=1}^{N} h_{i m} p_{i} \leq I_{\text {Thres }} \\
& 0 \leq p_{i} \leq P^{\max }, \quad i=1, \ldots, N
\end{array}
$$

The equations in (5) state the constraint introduced to guarantee a minimum SINR level for SU $i$ and to limit the interference level caused at the PU (i.e. the eNB) as well as the transmit power of each SU. Note that in this study, only one PU $(m=1)$ is considered.

\subsection{Convergence to the $\mathrm{NE}$}

The optimal transmit power or NE solution can be obtained in such a way that each SU maximizes his own utility function iteratively. The Lagrangian function of the convex equivalent of (4) is then:

$$
L\left(p_{i}, \lambda_{i}, \mu_{i}\right)=U_{i}\left(p_{i}\right)+\sum_{i=1}^{N} \lambda_{i}\left(\gamma_{i j}-\gamma_{\text {min }}\right)+\sum_{i=1}^{N} \mu_{i}\left(\sum_{i=1}^{N} h_{i m} p_{i}-I_{\text {Thres }}\right) \text { (6) }
$$

where $\lambda_{i}$ and $\mu_{i}$ are the Lagrangian multipliers.

Hence, the problem in (4) is equivalent to:

$$
\begin{aligned}
& \max _{p_{i}} L\left(p_{i}, \lambda_{i}^{*}, \mu_{i}^{*}\right) \\
& \text { s.t } \quad 0 \leq p_{i} \leq P^{\max } \text { for } i=1, \ldots, N
\end{aligned}
$$

The problem in (7) is solved via the following first-order algorithm that utilizes the gradient of $L\left(p_{i}, \lambda_{i}, \mu_{i}\right)$ to simultaneously update primal and dual variables with constant step size $\beta$ and $[\mathrm{x}]+=\max \{0, \mathrm{x}\}$ :

$$
\begin{aligned}
& p_{i}(k+1)=p_{i}(k)+\beta \frac{\partial L(p, \lambda, \mu)}{\partial p_{i}} \\
& \lambda_{i}(k+1)=\left[\lambda_{i}(k)+\beta \gamma_{i}\right]^{+} \\
& \mu_{i}(k+1)=\left[\mu_{1}(k)+\beta \sum_{i=1}^{N} h_{m i} p_{i}\right]^{+}
\end{aligned}
$$

The gradient $\nabla L\left(p_{i}, \lambda_{i}, \mu_{i}\right)$ is used in (6) to find the maximum of $L(p, \lambda, \mu)$ with respect to $p$, and convergence will lead to the NE.

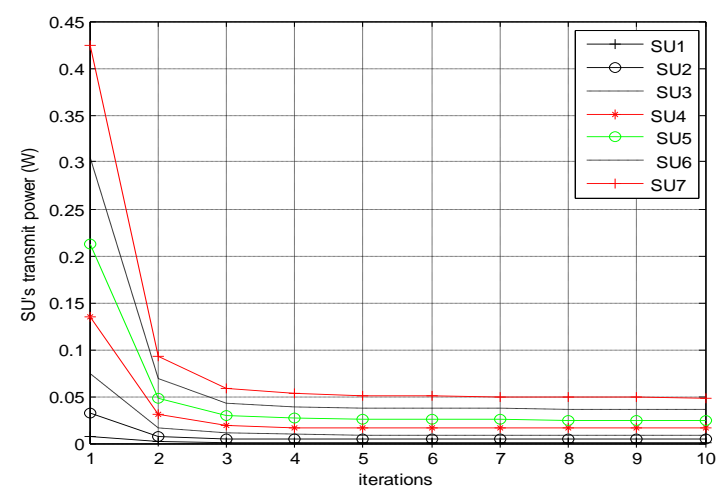

Figure2: Power allocation for SUs in the proposed noncooperative game 
Figure 2 shows the convergence of the transmit powers of seven SUs to a steady state. Thanks to the proposed noncooperative game, the obtained NE solution adapts the SUs' transmit powers by taking into account the different constraints in (5) and helps reduce the interference level in the network. Figure 3 shows indeed that the interference level measured at the PU (i.e. the eNB) is significantly reduced at the steady state (by almost 50\%) as compared to constant power allocation to SUs. In the latter, all SUs are supposed to transmit a power of $10 \mathrm{~mW}$ each. In the next section, cooperative solutions between the SUs are proposed to further enhance the system performance.

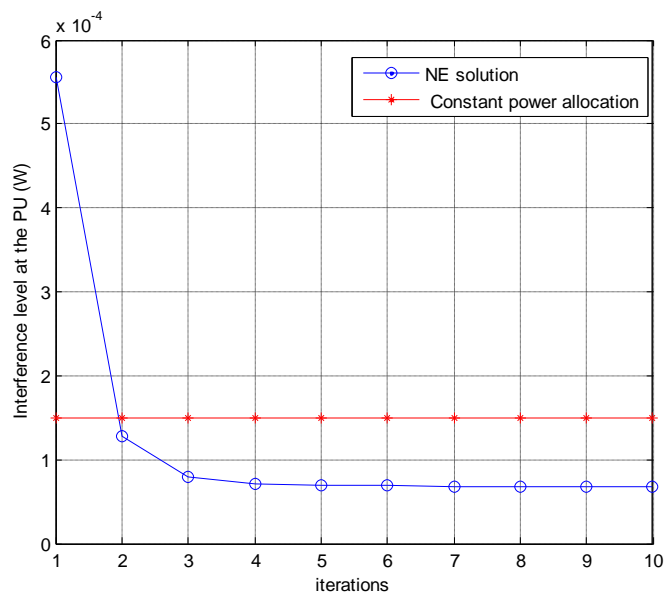

Figure 3: Estimated interference level at the PU

\section{PROPOSED COOPERATIVE BARGAINING SOLUTIONS}

In order to improve the efficiency and fairness of noncooperative games, usually considered as selfish games, and rather than grouping the SUs into coalitions (e.g. [21]), solutions based on Nash Bargaining games are adopted. Their inherent property of being proportionally fair [22] and Pareto optimal is indeed much desirable for an efficient distributed algorithm. Such cooperative games assume that a forcing agreement can be reached by the players of the game through a reciprocal information exchange. In this section, different bargaining solutions are established based on the noncooperative game SUs' payoffs given in eq.(3). More precisely, a comparison of the system performance of four different bargaining solutions is given: the Nash Bargaining Solution (NBS), Kalai-Smorodinsky Bargaining Solution (KSBS), the utilitarian solution (US) and the Egalitarian Solution (ES).

\subsection{Nash Bargaining Solution for Power Control Game}

The principle of a Nah Bargaining game is to share the resources between the players in order to maximize the overall users' payoff in the network. That's to maximize the Nash Product (NP). Given the SU payoff in (3), the NP is thus expressed as:

$$
N_{p}=\prod_{i=1}^{N}\left(U_{i}\left(p_{i}\right)-u_{i}^{0}\right)
$$

where $p_{i}$ is the power allocated to the SU $i . U_{i}$ is the utility of the SU $i$ when the $p_{i}$ is allocated to it. $U_{i}$ is the payoff of the $\mathrm{SU} i$ when no agreement is reached in the bargaining problem and $N$ is the number of SUs involved in the game. Let $u_{i}{ }^{0}$ be the disagreement point which corresponds to the minimum required payoff of the $\mathrm{SU} i$.

In order to solve the NBS, and thus reach the Pareto optimal, several conditions should be verified. Let $X^{*}=F\left(U_{i}, u_{i}^{0}\right)$ is said to be an NBS in $U_{i}$ for the disagreement point $u_{i}^{0}$, if the following Axioms are satisfied:

1. Individual Rationality: $X_{i}^{*} \geq u_{i}^{0}$ for all $i$.

2. Feasibility: $X \in S$.

3. Pareto Optimality: $X^{*}$ is Pareto optimal.

4. Independence of Irrelevant Alternatives: If $X \in S^{\prime} \subset S$ and $X^{*}=F\left(S, u_{i}^{0}\right)$, then $X^{*}=F\left(S^{\prime}, u_{i}^{0}\right)$.

5. Invariance to affine Transformations: For any linear scale transformation $\psi, \psi\left(F\left(S, u_{i}^{0}\right)=F\left(\psi(S), \psi\left(u_{i}^{0}\right)\right)\right.$.

6. Symmetry: If $S$ is invariant under all exchanges of users, $F_{i}\left(S, u_{i}^{0}\right)=F j\left(S, u_{i}^{0}\right)$ for all possible players $i, j$.

The Axioms 1, 2 and 3 represent the binding conditions for any agreement point given by a bargaining solution, while the Axioms 4, 5 and 6 represent the fairness Axioms. The symmetry axiom states that if the feasible set is completely symmetric for all players and they have the same disagreement point then the NBS is the same for all players.

Since the logarithm is a continuous strictly increasing function, solving the problem in (9) is equivalent to finding the solution of the following problem:

$$
\begin{aligned}
& \ln \left(\max \left(\prod_{i=1}^{N}\left(U_{i}\left(p_{i}\right)-u_{i}^{0}\right)\right)\right)=\max \ln \left(\prod_{i=1}^{N}\left(U_{i}\left(p_{i}\right)-u_{i}^{0}\right)\right) \\
& =\max \sum_{i=1}^{N} \ln \left(U_{i}\left(p_{i}\right)-u_{i}^{0}\right)
\end{aligned}
$$$$
\text { s.t } \quad \sum_{i=1}^{N} h_{i m} p_{i} \leq I_{\text {Thres }}
$$

$$
0 \leq p_{i} \leq P^{\max }, \quad i=1, \ldots, N
$$

The problem in (10) is equivalent to the problem in (9) since the feasibility set is convex and compact, and the utility functions are injective and strictly concave. Therefore, it can be solved using convex programming methods as Lagrange multipliers method. To solve (9), Lagrange multiplier method is used. The cost function $L$ is written as follows:

$$
\begin{aligned}
& L\left(p_{i}, \lambda_{i}, \mu_{i}\right)=\sum_{i=1}^{N} \ln \left(U_{i}\left(p_{i}\right)-u_{i}^{0}\right)+\sum_{i=1}^{N} \lambda_{i}\left(p_{i}-P^{\max }\right) \\
& +\sum_{i=1}^{N} \mu_{i}\left(\sum_{i=1}^{N} h_{i m} p_{i}-I_{\text {Thres }}\right)
\end{aligned}
$$

The problem (10) is equivalent to:

$$
\begin{aligned}
& \max _{p_{i}} L\left(p_{i}, \lambda_{i}^{*}, \mu_{i}^{*}\right) \\
& \text { s.t } \quad p_{i} \geq 0 \text { for } i=1, \ldots, N
\end{aligned}
$$


where $\lambda_{i}$ and $\mu_{i}$ are the Lagrange multipliers. The problem in (13) is solved by using the gradient of $L\left(p_{i}, \lambda_{i}, \mu_{i}\right)$, finding the partial derivative of $L$ in $p_{i}$ and resolving both derivatives equal to 0 .

Figure 4 illustrates the convexity of the Nash Product function which derived in case of a scenario in which two D2D Tx users bargain their transmit power in order to communicate simultaneously with other cellular users.

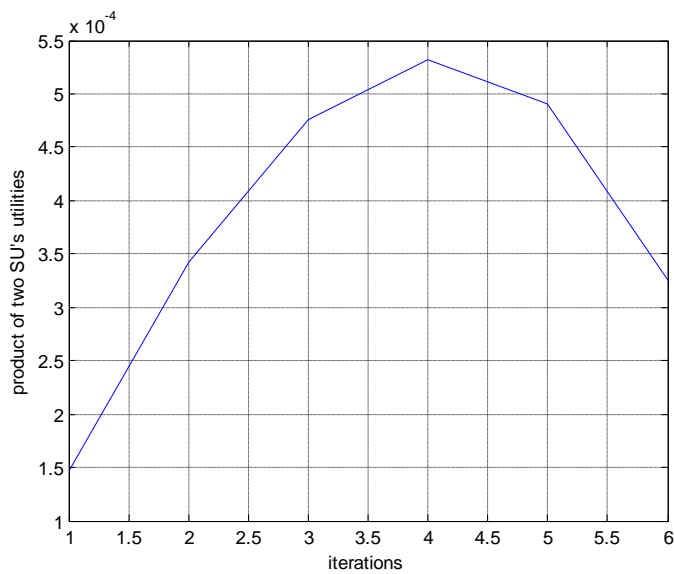

Figure 4 : Nash Product Function of two pairs of SUs

\subsection{Kalai-Smorodinsky Bargaining Solution}

Another bargaining solution is considered as alternative to NBS is the Kalai-Smorodinsky Bargaining Solution (KSBS). First, an explanation of the fundamentals of the KSBS is given [23], and then applied to the power allocation problem for cognitive $\mathrm{D} 2 \mathrm{D}$ communications.

A bargaining problem is defined as a pair $\left(U=\left(u_{1}, \ldots, u_{N}\right), d\right)$, where $U \subset R$. $U$ is the set of feasible payoffs and $d \in U$ is the disagreement point.

With $B$ denoting the set of all possible bargaining problems, a bargaining solution is a function $f: B \rightarrow U$. KSBS is the solution which satisfies four axioms: Pareto optimality, symmetry, invariance to affine transformations and individual monotonicity [22].

If $U$ is convex and compact, the KSBS must satisfy $u^{\max }$, called the "utopia" point, denotes the maximal achievable payoff. Achieving $u_{i}^{\max }$ corresponds to allowing a SU $i$ to occupy all resources. Thus, the KSBS assigns as the bargaining solution the point in the boundary of feasible set that intersects the line connecting the disagreement point and the utopia point. The mathematical representation of the KSBS is the point defined as:

$\left(u_{1}^{*}, u_{2}^{*}\right)=\arg \max \left\{u>d \cap \frac{u_{1}-d_{1}}{u_{1}^{\max }-d_{1}}=\frac{u_{2}-d_{2}}{u_{2}^{\max }-d_{2}}\right\}$ (14)

\subsection{Other Proposed Bargaining Solutions}

The third bargaining solution considered in this work, is the Egalitarian Solution (ES). The latter represents the point in the feasible set where all players achieve maximal equal increase in utility with respect to the disagreement point $d$ [23]. According to this definition, the ES must be the intersection of the line $x=y$ and the Pareto optimal curve $P$. It is also mathematically defined as: $\left(u_{1}^{*}, u_{2}^{*}\right)=\arg \max \left\{u>d \cap u_{1}-d_{1}=u_{2}-d_{2}\right\}$

The last bargaining solution considered in this paper is the Utilitarian Solution (US). This solution maximizes the sum of the utilities and is not always unique. The US is the point where $x+y=a$ is tangential to $P$ [23], where each player gain is proportional to its maximum utility. This also maximizes the $\operatorname{sum}(x+y)$. The US is mathematically represented by:

$$
\left(u_{1}^{*}, u_{2}^{*}\right)=\arg \max \left\{u>d \cap \frac{u_{1}-d_{1}}{u_{1}^{\max }}=\frac{u_{2}-d_{2}}{u_{2}^{\max }}\right\}
$$

\subsection{Comparison between the different bargaining solutions}

Figure 5 illustrates a geometrical representation of a twoplayer bargaining game. Along the $\mathrm{x}$-axis and $\mathrm{y}$-axis are the payoff for the first and second player; respectively, a closed convex region representing the feasible set is formed, the boundary, which is called Pareto optimal curve $P$, is where bargaining solutions should rest on.

Figure 5.(a) shows the NBS solution which is the point on the Pareto boundary where the line from the origin to NBS makes the same angle to the horizontal as the tangent line to the feasible set. The figure 5.(b) represents the US which is the tangent point of the line at $\arctan (\mathrm{u} 1 \max / \mathrm{u} 2 \max )$ and the curve P. Figure5.(d) represents the KSBS solution which is the intersection of the line joining the utopia point to the disagreement point $d$, and the Pareto boundary. Finally, the ES is illustrated on the figure 5.(c) as the intersection of the $45^{\circ}$ line and the Pareto boundary.
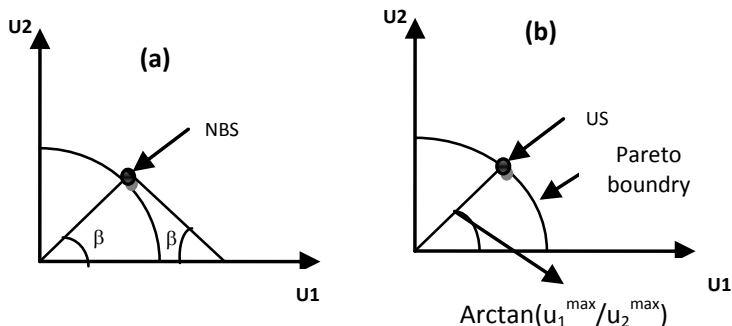

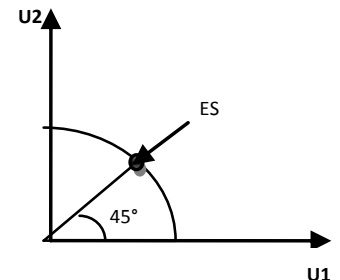

(c)

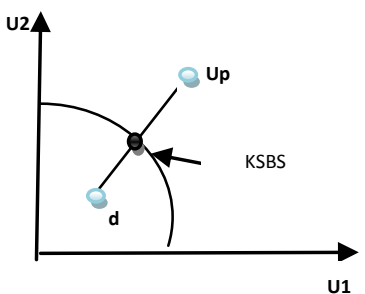

(d)
Figure 5: Geometric representation of bargaining solutions of a game

\section{PERformance eVAluation}

In this section, the above proposed algorithms are run with Matlab and the system performances are evaluated assuming the proposed utility function of the different bargaining games. Simulation parameters are given in Table I.

The results of the NBS are compared to the corresponding results of the KSBS, US and ES. They are also compared to the performances of the non-cooperative solution (NE). 
Table1. Simulation Parameters

\begin{tabular}{|l|l|}
\hline \multicolumn{1}{|c|}{ Parameter } & \multicolumn{1}{c|}{ Value } \\
\hline Bandwidth & $5 \mathrm{Mhz}$ \\
\hline Cell Radius & $500 \mathrm{~m}$ \\
\hline Channel bandwidth $W$ & $10 \mathrm{MHz}$ \\
\hline PU Transmit Power & $10 \mathrm{~W}$ \\
\hline $\begin{array}{l}\text { Maximum SU Transmit Power } \\
P_{\max }\end{array}$ & $1 \mathrm{~W}$ \\
\hline ITL at the PU & $10^{-10} \mathrm{~W}$ \\
\hline Noise power density & $-174 \mathrm{dBM} / \mathrm{Hz}$ \\
\hline Pathloss between SU and PU & $140.7+36.7 \log 10\left(d_{\mathrm{sp}}\right)$ \\
\hline $\begin{array}{l}\text { Pathloss between a SU Tx and a } \\
\text { SU Rx }\end{array}$ & $130.62+37.6 \mathrm{x} \log _{10}\left(d_{s s}\right)$ \\
\hline Log-normal shadowing fading & $10 \mathrm{~dB}$ \\
\hline
\end{tabular}

\subsection{Convergence to the Pareto-Optimal point}

The bargaining solutions of games are considered and only two pairs of D2D users negotiate their transmit power allocation. Figure 6 illustrates the respective utilities $U_{1}$ and $U_{2}$. The shaded region represents the feasible set of utilities, it also includes the point $\left(\mathrm{u}_{1}{ }^{\mathrm{NE}}, \mathrm{u}_{2}{ }^{\mathrm{NE}}\right)$, which is the pure NE. Figure 6 also shows that the different bargaining solutions NBS, KSBS, ES and US are optimal as they are located on the Pareto optimal boundary, whereas the non-cooperative solution (NE) is not an optimal solution.

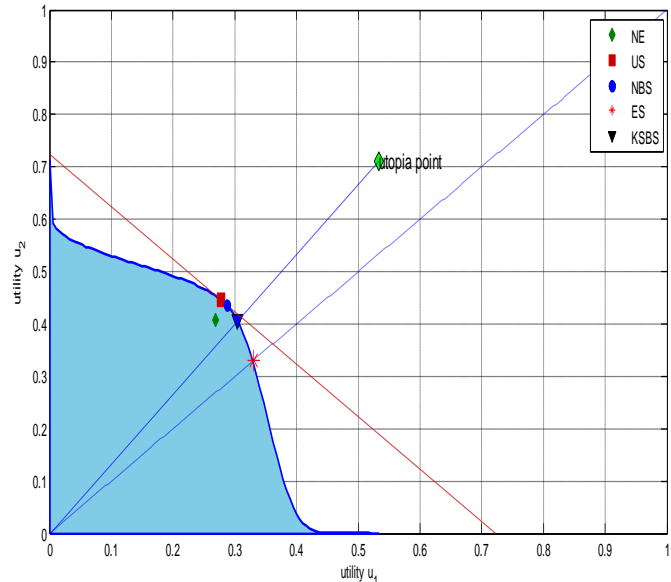

Figure 6: Different bargaining solutions Pareto-optimal convergence

\subsection{Energy Efficiency and Fairness \\ Evaluation}

Recent researches target to improve the energy efficiency of emerging $5 \mathrm{G}$ networks by a factor of 1000 per transported bit. Let $\eta$ be the Energy Efficiency (EE) function. This function, consisting of the ratio of the total radio capacity of all SUs to their total transmit powers, is given by:

$$
\eta=\frac{\sum_{i=1}^{N} R_{i}}{\sum_{i=1}^{N} p_{i}}
$$

Figure 7 illustrates the EE of the different bargaining solutions as well as the non-cooperative one. The US always achieves optimal value of the EE, as it maximizes total SU's throughput. The US provides nearly $40 \%$ improvements over the NBS and KSBS in terms of throughput. It also shows that the NBS and the KSBS solutions achieve approximately $3 \mathrm{Mb} / \mathrm{J}$, whereas the NE solution often gives results far from optimum, nearly $1.5 \mathrm{Mb} / \mathrm{W}$. Hence, the US and the NBS solutions enable the cognitive D2D Tx to communicate in an energy efficient manner.

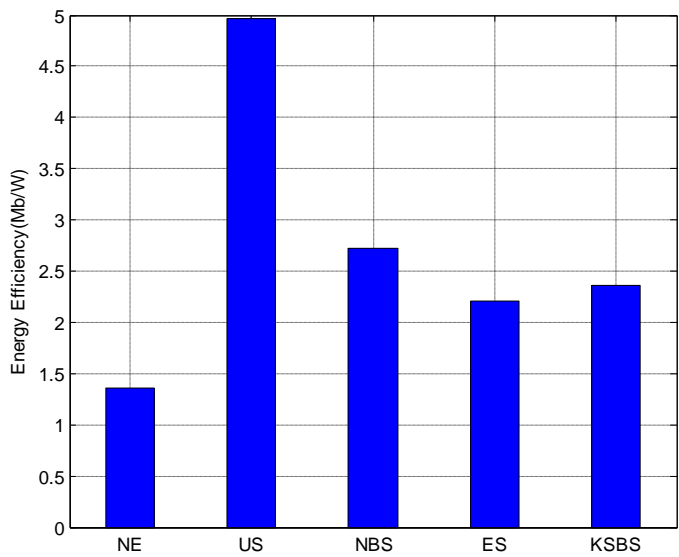

Figure 7 : Comparison of Energy Efficiency for the different bargaining solutions

A numerical evaluation has been performed in Matlab, aiming at understanding how the proposed bargaining solutions answer to the needs of the fairness in the proposed solutions.

Jain's fairness index which is defined in [12] by the function:

$$
F I(U)=\frac{\left(\sum_{i=1}^{N} u_{i}\right)^{2}}{N \sum_{i=1}^{N} u_{i}{ }^{2}}
$$

where the achieved utility is used as the measurement metric, and $0<\mathrm{FI}(U)<1$. Jain's index is one of the earliest proposed and widely studied fairness measures. A large value of $\operatorname{FI}(U)$ represents fairer resource allocation from the system perspective.

Figure 8 shows the different Jain's index values of the proposed bargaining solutions. In this figure, the allocation tends to be fairer when Jain's index is closer to 1 . It is observed that both NBS and ES solutions maintain good proportional fairness among players, whereas the NE solution has a very poor fairness index. As it clearly appears, the KSBS has lower fairness index value (FI $(U)=0.6)$ than the US which presents a $\operatorname{FI}(U)=0.9$ and the NBS and ES which reach a value very close to 1 . This result is expected, since the US is maximizing the total throughput, whereas the ES guarantees equal increase in the utility distribution, and the NBS has the property of proportional fairness. 


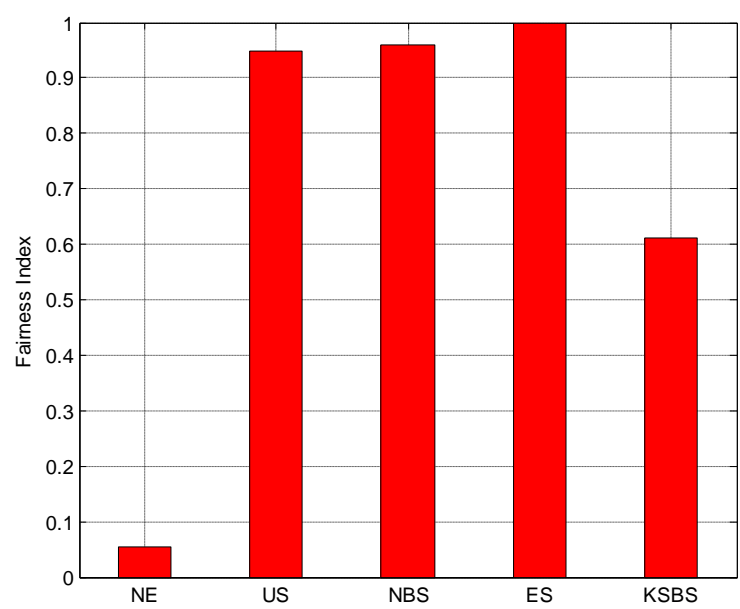

Figure 8: Comparison of the Fairness Index for the different bargaining solutions

Figure 7 and 8 also show that cooperative solutions are more fair and energy efficient than the non-cooperative solution.

\section{CONCLUSION}

In this paper, the problem of power allocation is studied in a cognitive D2D communications. D2D communications form indeed one key application of the $5 \mathrm{G}$ wireless system, which can enable devices in proximity to communicate directly bypassing the eNB through cognitive capabilities. This study aims at helping mitigate the interference of cognitive D2D transmitters at the eNB, and find a fair and energy efficient method to allocate power among cognitive D2D devices. First, a non-cooperative game proposed between the cognitive D2D Tx users and takes into account the interference level at the cellular base station. Its convergence to a steady state is proved. Then, different cooperative bargaining games are examined based on the former users' utilities. The geometric interpretation of the resulting bargaining solutions is given by providing the Pareto-optimal point by simulations. It is also proved that NBS achieves better performance in terms of energy efficiency and fairness than non-cooperative game and other cooperative bargaining solutions such as KSBS, ES and US. This study can be extended to other emerging $5 \mathrm{G}$ applications like M2M networks, Infrastructure of Vehicles (IoV) and IoT.

\section{REFERENCES}

[1] Li, H., Gai, Y., He, Z., Niu, K., and Wu, W., 2008, Optimal power control game algorithm for cognitive radio networks with multiple interference temperature limits," in Proceedings of IEEE Vehicular Technology Conference, pp. 1554-1558, May 2008.

[2] IEEE, 2005, Standard 802.16e-2005. part16: Air interface for fixed and mobile broadband wireless access systems amendment for physical and medium access control layers for combined fixed and mobile operation in licensed band.

[3] Magri H., Abghour N. and Ouzzi M., 2016, Device toDevice (D2D) communications under LTE-Advanced networks, International Journal of Wireless \& Mobile Networks (IJWMN) Vol. 8, No. 1, February 2016

[4] Doppler, K., Rinne, M., Wijting, C., Ribeiro, C. and Hugl K., 2009, Device to-Device Communication as an
Underlay to LTE-Advanced Networks, IEEE Communications Magazine, vol. 47, pp. 42-49, Dec. 2009.

[5] Third Generation Partnership Project (3GPP), "Physical layer procedures (Release 10) for Evolved Universal Terrestrial Radio Access (EUTRA)," 3GPP TS $36.213 \mathrm{v}$ 10.5.0, 2012.

[6] Cheng, P., Deng, L., Yu, H., Xu, Y., Wang, H., 2012 , Resource Allocation for Cognitive Networks with D2D Communication: An Evolutionary Approach, 2012 IEEE Wireless Communications and Networking Conference: Mobile and Wireless Networks.

[7] Alireza, A., Nakhai, M. R., and Hamid, A. A., 2009 "Cognitive radio game for secondary spectrum access problem," IEEE Trans. Wireless Commun., vol. 8, no. 4, pp. 2121-2131, Apr. 2009.

[8] Rasti, M., Sharafat, A. R., and Seyfe, B., Pareto-efficient and goal-driven power control in wireless networks: A game-theoretic approach with a novel pricing scheme, IEEE/ACM Trans. Netw., vol. 17, no. 2, pp. 556- 569, Apr. 2009.

[9] Song, L., Niyato, D., Han, Z., and Hossain, E., 2014, Game-theoretic Resource Allocation Methods for Device-to-Device (D2D) Communication. IEEE Wireless Communications, Volume: 21, Issue: 3, June 2014 .

[10] Cheng P., Deng L., Yu H., Xu Y., Wang H., 2012, Resource Allocation for Cognitive Networks with D2D Communication: An Evolutionary Approach, IEEE Wireless Communications and Networking Conference: Mobile and Wireless Networks.

[11] Gorni, Del Re E., Ronga, G., Suffritti, L. R., 2009, A power allocation strategy using Game Theory in Cognitive Radio networks, in Proceedings of the First ICST international conference on Game Theory for Networks, GameNets'09, pp. 117 - 123 ，13-15 May 2009.

[12] Jing, Q. and Zheng, Z. , 2009 , Distributed Resource Allocation Based on Game Theory in Multi-cell OFDMA Systems, International Journal of wireless information networks, Volume 16, Numbers 1-2, 44-50. March 2009.

[13] Guan, Z., Yuan, D., Zhang, H., and Ding, L., 2014 Cooperative bargaining solution for efficient and fair spectrum management in cognitive wireless networks, International Journal of Communication Systems, Volume 27, Issue 11, pages 3441-3459, November 2014

[14] Ni, Q., Zarakovitis Charilaos, C., 2012,Nash Bargaining Game Theoretic Scheduling forJoint Channel and Power Allocation in Cognitive Radio Systems. IEEE Journal on selected areas in communications, VOL. 30, NO. 1, January 2012

[15] Yang, C., Li, J.D., and Tian, Z., 2010, “Optimal Power Control for Cognitive Radio Networks Under Coupled Interference Constraints: A Cooperative Game-Theoretic Perspective", IEEE transactions on Vehicular Technology, VOL. 59, NO. 4, May 2010

[16] Asadi, A., Wang, Q., and Mancuso, V., 2014, A Survey on Device-to-Device Communication in Cellular 
Networks," IEEE Communications Surveys \& Tutorials, vol. 16, no. 4, pp. 1801-1819, Nov. 2014.

[17] Wang, F., Song, L., Han, Z., Zhao, Q., and Wang X., 2013, Joint scheduling and resource allocation for device-to-device underlay communication, IEEE Wireless Communications and Networking Conference (WCNC), Shanghai, China, Apr. 2013

[18] Wang, F., Xu, C., Zhao, Q., Wang, X., and Han, Z., 2013, Energy-aware resource allocation for device-todevice underlay communication," IEEE International Conference on Communications (ICC), Budapest, Hungary, Jun., 2013.

[19] Song, L., Niyato, D., Han, Z., and Hossain E., 2014, Game-theoretic Resource Allocation Methods for Device-to-Device (D2D) Communication, IEEE Wireless Communications Magazine, vol. 21, no. 3, pp. 136-144, Jun. 2014.
[20] Yu, C.-H., Doppler, K., Ribeiro, C., and Tirkkonen, O., 2011, Resource sharing optimization for D2D communication underlaying cellular networks, IEEE Trans. Wireless Commmun., vol. 10, no. 8, pp. 2752 2763, Aug. 2011.

[21] Hamouda, S., El-Bessi, S., Tabbane, S., 2014, New Coalition Formation Game for Spectrum Sharing in Cognitive Radio Networks, International Journal of Communication Systems, 2014.

[22] Yaacoub, E., Dawy, Z., 2011, Achieving the Nash bargaining solution in OFDMA uplink using distributed scheduling with limited feedback, Elsevier 2011

[23] Militano, L. ; Condoluci, M. ; Araniti, G. ; Iera, A., Bargaining Solutions for Multicast Subgroup Formation in LTE, Vehicular Technology Conference (VTC Fall), 2012 IEEE 